\title{
Desenvolvimento e avaliação da usabilidade de um aplicativo para registro eletrônico de atendimento pré-hospitalar
}

Development and evaluation of the usability of a mobile application for electronic registration of pre-hospital emergency care

Desarrollo y evaluación de la usabilidad de una aplicación móvil para el registro electrónico de la atención de emergencias prehospitalarias

Edmara Aparecida Francisco Augusto

ORCID: https://orcid.org/0000-0002-7961-1861 Faculdade de Medicina de São José do Rio Preto, Brasil E-mail: edmarafr@gmail.com

Alexandre Lins Werneck

ORCID: https://orcid.org/0000-0002-2911-8091 Faculdade de Medicina de São José do Rio Preto, Brasil E-mail: alexandre.werneck@famep.br

Luciene Cavalcanti Rodrigues

ORCID: https://orcid.org/0000-0002-3528-5574 Instituto Federal de Educação, Ciência e Tecnologia de São Paulo, Brasil E-mail: prof.luciene@fatecriopreto.edu.br

Rita de Cássia Helú Mendonça Ribeiro

ORCID: https://orcid.org/0000-0002-1016-0484 Faculdade de Medicina de São José do Rio Preto, Brasil E-mail: ricardo.rita@terra.com.br

Vânia Del' Arco Paschoal

ORCID: https://orcid.org/0000-0002-6047-5345 Faculdade de Medicina de São José do Rio Preto, Brasil E-mail: vaniadelarco@gmail.com

Daniele Alcalá Pompeo

ORCID: https://orcid.org/0000-0003-2671-2586 Faculdade de Medicina de São José do Rio Preto, Brasil E-mail: daniele.pompeo@famerp.br

\begin{abstract}
Resumo
O presente artigo descreve o processo de desenvolvimento e validação de um aplicativo para registro eletrônico de atendimento pré-hospitalar. Trata-se de um estudo de avaliação tecnológica de corte transversal com delineamento descritivo, abordagem quantitativa do tipo analítica com correlação entre variáveis. O aplicativo foi construído pela pesquisadora seguindo as fases de engenharia de software: análise de requisitos, projeto e especificação, construção, testes internos, manutenção e avaliação externa. O aplicativo foi avaliado utilizando o instrumento System Usability Scale (SUS). A amostra foi composta por 25 participantes: 15 profissionais de atendimento pré-hospitalar (APH), enfermeiros e técnicos de enfermagem que atuam no SAMU de São José do Rio Preto e 10 profissionais de Tecnologia da Informação (TI). Conforme resultado da validação, o aplicativo APH Mobile atende aos critérios de facilidade de aprendizagem do sistema $(77,92) \pm 0,09$, eficiência do sistema $(75,76) \pm 0,03$, facilidade de memorização $(66,67)$ minimização dos erros $(76,67)$ e satisfação dos usuários $(84,44) \pm 0,06$. A pontuação SUS do aplicativo foi de $76,67 \pm$ 17,57 demonstra que o aplicativo atende exigências de usabilidade. $\mathrm{O}$ aplicativo tem a capacidade de aumentar a eficiência do processamento das informações, por meio de uma coleta de dados mais imediata, possibilitando análise dos dados e resultando em ganhos assistenciais e gerenciais, decorrentes da disponibilidade de informações abrangentes, precisas e a tempo.
\end{abstract}

Palavras-chave: Aplicativos móveis; Registro eletrônico de saúde; Atendimento de emergência pré-hospitalar.

\begin{abstract}
:
The article describes the developing and validating process of a mobile application for pre-hospital emergency care electronic registration. We carried out a cross-sectional technological assessment study, using a descriptive design and a quantitative analytical approach. The researcher built the mobile application following the software engineering phases: requirements analysis, design and specification, construction, internal testing, maintenance, and external evaluation. We use the System Usability Scale (SUS) instrument to evaluate the mobile application. The study sample consisted of 25 participants: 15 pre-hospital care (PHC) professionals, registered nurses, licensed practical nurses
\end{abstract}


working at the SAMU in São José do Rio Preto, and 10 Information Technology (IT) professionals. As a result of the validation, the APH Mobile application met the criteria of ease of learning the system $(77.92) \pm 0.09$, system efficiency $(75.76) \pm 0.03$, ease of memorization (66.67), minimization errors (76.67), and user satisfaction (84.44) \pm 0.06 . The application's SUS score of $76.67 \pm 17.57$ demonstrated that the application meets usability requirements. The application can increase the efficiency of information processing through a more immediate data collection, enabling data analysis and resulting in assistance and management gains, resulting from the availability of comprehensive, accurate, and timely information.

Keywords: Mobile applications; Electronic health records; Pre-hospital emergency care.

\section{Resumen}

El artículo describe el proceso de desarrollo y validación de una aplicación móvil para el registro electrónico de atención de emergencia prehospitalaria. Realizamos un estudio de evaluación tecnológica transversal, con un diseño descriptivo y un enfoque analítico cuantitativo. El investigador construyó la aplicación móvil siguiendo las fases de ingeniería de software: análisis de requisitos, diseño y especificación, construcción, pruebas internas, mantenimiento y evaluación externa. Usamos el instrumento System Usabilidad Scale (SUS) para evaluar la aplicación móvil. La muestra del estudio estuvo formada por 25 participantes: 15 profesionales de la atención prehospitalaria (APS), enfermeras registradas, enfermeras prácticas licenciadas que trabajan en el SAMU en São José do Rio Preto y 10 profesionales de Tecnologías de la Información (TI). Como resultado de la validación, la aplicación APH Mobile cumplió con los criterios de facilidad de aprendizaje del sistema $(77,92) \pm 0,09$, eficiencia del sistema $(75,76) \pm 0,03$, facilidad de memorización $(66,67)$, minimización de errores $(76,67)$ y satisfacción del usuario. $(84,44) \pm 0,06$. La puntuación SUS de la aplicación de 76,67 \pm 17,57 demostró que la aplicación cumple con los requisitos de usabilidad. La aplicación puede aumentar la eficiencia del procesamiento de la información a través de una recopilación de datos más inmediata, lo que permite el análisis de datos y da como resultado ganancias de asistencia y administración, como resultado de la disponibilidad de información completa, precisa y oportuna.

Palabras clave: Aplicaciones móviles; Registros electrónicos de salud; Atención de emergencia prehospitalaria.

\section{Introdução}

O Ministério da Saúde define a assistência prestada no nível primário de atenção aos pacientes portadores de quadros agudos, de natureza clínica, traumática ou ainda psiquiátrica, que possa levar a sofrimento, sequelas ou mesmo à morte, como Atendimento Pré-hospitalar (APH) (Portaria 288, 2018). Quando o paciente é atendido, esse atendimento pré-hospitalar gera um formulário de registro do atendimento com os dados de identificação do paciente, condições clinicas, e tratamento efetuado, preenchidos pelo profissional que presta o socorro (Ribeiro, 2017).

As equipes de salvamento no cenário do evento, coletam e registram os dados na ficha de atendimento, pois representam a única fonte de informação a respeito do atendimento. É um documento legal e exige informações que descrevam a avaliação realizada e os cuidados prestados, representando, assim, o prontuário do paciente para o atendimento pré-hospitalar (Gorini, 2018). Não há, por parte do Ministério da Saúde, qualquer indicação quanto ao padrão de preenchimento e elaboração dos registros da ficha de atendimento. Portanto, cada município é responsável pela elaboração do seu instrumento de atendimento pré-hospitalar, bem como, dos seus serviços de urgência e emergência (Barros et al., 2014).

$\mathrm{Na}$ análise documental de prontuários de pacientes, nos reduzidos artigos científicos que abordam sobre a urgência préhospitalar, destaca-se a riqueza de informações e a possibilidade da informatização do prontuário, mas também evidencia a fragilidade associadas, principalmente as falhas humanas em decorrência da negligência nos registros e anotações (Santana et al., 2019) e, chamam a atenção, os resultados que indicam o alto percentual de profissionais que ignoram dados na ficha de atendimento (Barros et al., 2014). Os registros eletrônicos estão substituindo os de papel, de forma irreversível. A informatização corresponde a um avanço significativo nos processos de trabalho, possibilitando melhor organização, sistematização e comunicação das informações, e os profissionais e instituições da área da saúde vêm incorporando tecnologias digitais para anotações de atividades do dia a dia (Lima et al., 2018).

Mediante a avaliação e análise dos dados registrados pelos serviços, por meio de informações contidas na ficha de atendimento pré-hospitalar, se estabelecem subsídios para elaboração de estratégias e programas de políticas públicas em urgência. O registro das informações, realizado na área de saúde, é de extrema relevância, uma vez que se demonstra a evolução 
do paciente assistido e, consequentemente, direciona o melhor processo terapêutico ou reabilitação, viabilizando um atendimento qualificado (Albuquerque et al., 2017).

A Tecnologia da Informação e Comunicação (TIC), pode disponibilizar maior facilidade na realização dos trabalhos e incrementar a assistência prestada, visto que possuem dispositivos que estruturam e organizam dados institucionais, os quais possibilitam o armazenamento, processamento, compartilhamento e acesso em tempo real ou remoto, que tem a finalidade de solucionar as necessidades em saúde (Gomes et al., 2018). Os serviços da saúde vêm automatizando seus processos sejam eles operacionais, administrativos ou assistenciais, na tentativa de alcançar uma melhoria na qualidade do atendimento, aumento da eficiência e, principalmente, na segurança dos dados do paciente, amparando tecnologicamente todos os procedimentos na área da saúde (Albuquerque et al., 2017).

No atendimento de emergência pré-hospitalar, a rapidez, a disponibilidade de informações e a facilidade de acesso são fatores imprescindíveis (Bernardi et al., 2018). Entendendo que o uso da tecnologia pode facilitar esta atividade, decidiu-se desenvolver um aplicativo para registro de atendimento pré-hospitalar (APH). Os objetivos foram desenvolver um aplicativo para dispositivo móvel para ser utilizado para registro das ocorrências atendidas e avaliá-lo quanto à satisfação do usuário e usabilidade.

\section{Metodologia}

Realizamos uma pesquisa aplicada, de estudo de produção e avaliação tecnológica de corte transversal com delineamento descritivo, abordagem quantitativa do tipo analítica, com correlação entre variáveis. No método quantitativo, a coleta de dados quantitativos ou numéricos é por meio do uso de medições de grandezas e é possível obter por meio da metrologia, números com suas respectivas unidades. Com isso, geram conjuntos de dados que podem ser analisados por meio de técnicas matemáticas (Pereira, et al., 2018).

O desenvolvimento do software foi baseado no ciclo de vida de desenvolvimento de sistemas (Pressman, 2016). As etapas foram (a) comunicação, na qual se realizou o levantamento dos requisitos do software; (b) planejamento, em que se descreveu os recursos que seriam utilizados e o cronograma a ser seguido; (c) modelagem que executou a criação do modelo consistente com requisitos levantados; (d) construção que combinou a geração de códigos e testes para revelar erros e, finalmente, (e) implantação que foi a etapa final, na qual o produto é analisado e avaliado (Barra et al., 2017).

Para o desenvolvimento do aplicativo, foi escolhido o framework IONIC, que permite a criação de aplicativos móveis híbridos. Optou-se por utilizar um framework híbrido, em função da necessidade de o aplicativo estar disponível pelos sistemas operacionais iOS e Android. Para armazenagem dos dados cadastrados pelo aplicativo, foi utilizado o Realtime Database, do Firebase, que permite o armazenamento e sincronismo dos dados entre usuários e dispositivos em tempo real com um banco de dados NoSQL (é um termo que representa os bancos de dados não relacionais.) hospedado na nuvem. Além disso, os dados permanecem disponíveis caso o aplicativo fique off-line, o que oferece uma ótima experiência do usuário, independentemente da conectividade de rede (Orlandi, 2019).

Atendendo ao segundo objetivo, a avaliação do aplicativo ocorreu com dois grupos diferentes: profissionais da saúde e profissionais de computação. Os profissionais de computação foram convidados de acordo com o seguinte critério: ter preferencialmente, no mínimo, dois anos de experiência na área. A escolha dos profissionais de saúde teve os seguintes critérios de inclusão: enfermeiros que atuam no SAMU de São de José do Rio Preto, e dois técnicos de enfermagem de cada plantão, totalizando dezenove pessoas convidadas. Foram excluídos os profissionais que estavam de licença ou em gozo de férias.

As avaliações ocorreram entre os meses de setembro e outubro de 2019. Assim, ao final do período da coleta da pesquisa, foram obtidas 25 participações, sendo 10 profissionais em computação, e 15 profissionais de saúde que atuam no atendimento pré-hospitalar (técnicos e enfermeiros). Inicialmente, os profissionais de saúde que atuam no atendimento pré- 
hospitalar, receberam os vídeos explicativos para instalação do aplicativo nos seus aparelhos e apresentação das principais funcionalidades contidas no aplicativo. Os dois grupos de participantes, receberam um estudo de caso com a finalidade de simular a prática de registro do atendimento diretamente no aplicativo. O estudo de caso foi enviado em arquivo em formato PDF, por meio do aplicativo de troca de mensagens. O estudo de caso recebido pelos profissionais da computação, não continha termos específicos da área de saúde. De posse do caso, o avaliador utilizava o aplicativo para registrar o atendimento realizado. Não foi cronometrado o tempo do registro do atendimento pelos participantes.

Para avaliação de satisfação dos usuários, foi aplicado o questionário System Usability Scale (SUS), um dos mais aceitos pela confiabilidade e validade (Bangor, et al., 2008). O SUS contém uma escala simples de dez questões, possibilitando avaliação de percepções subjetivas. As dez questões pertencentes ao SUS, são: 1- Acho que gostaria de usar esse sistema com frequência. 2- Achei o sistema muito complexo. 3- Achei o sistema fácil de usar. 4- Achei que seria necessário o apoio de um técnico para poder usar este Sistema. 5- As funções do sistema estão muito bem integradas. 6- Achei que o sistema apresenta muita inconsistência. 7- Imagino que a maioria das pessoas aprenderiam a usar esse sistema rapidamente. 8- Achei o sistema muito complicado de usar. 9- Me senti confiante ao usar o sistema. 10- Eu preciso aprender várias coisas antes de continuar usando esse sistema (Nielsen, 2017).

O instrumento SUS, utiliza escala Likert com valores 1 (discordo plenamente) a 5 (concordo plenamente), em que 3 significa neutro. Para calcular a pontuação SUS, são somadas as contribuições de cada item de pontuação. Para os itens ímpares $(1,3,5,7$ e 9), a contribuição da pontuação é a posição da escala menos 1, para os itens pares (4, 6, 8 e 10), a contribuição é de 5 menos a posição da escala. Somam-se as contagens do total das 10 questões e multiplica-se por 2,5 para obter o valor global de usabilidade do sistema. As pontuações do SUS têm uma gama de 0 a 100, sendo que menor que 51 é considerado ruim, maior que 71 é bom, maior que 86 é excelente e maior que 91 é o melhor alcançável (Bangor, et al., 2009). Este questionário foi empregado em outros estudos, o que também contribuiu para que fosse escolhido (Tenório, 2011). Em um estudo se equiparou o SUS Score a valores relativos, a mediana foi determinada em 68, indicando que para valores acima 68 está superior da média e inferior 68 está abaixo da média. (Bangor, 2008). Diante da pontuação encontrada, foi possível fazer a classificação do sistema avaliado.

A usabilidade é um atributo de qualidade que avalia a facilidade de uso de uma interface, sendo definida por cinco componentes: 1. Capacidade de aprendizagem: a facilidade de utilizar o sistema pela primeira vez; 2. Eficiência: rapidez para executar as tarefas; 3. Memorização: o processo de lembrar como utilizar o sistema, após um tempo sem utilizar; 4. Erros: ausência de erros apresentados pelo sistema; 5. Satisfação: design agradável (Tenório, 2011). É possível reconhecer os componentes de qualidade indicados por Nielsen nas questões do SUS: • Facilidade de aprendizagem: 3, 4, 7 e 10; • Eficiência: 5, 6 e 8; • Facilidade de memorização: 2; • Minimização dos erros: 6; • Satisfação: 1, 4, 9 (Bangor, 2009).

Esta pesquisa foi aprovada pelo Comitê de Ética e Pesquisa da Faculdade de Medicina de São José do Rio Preto (FAMERP), Parecer $\mathrm{n}^{\circ}$ 3.581.901. A participação na pesquisa foi mediante aceite do Termo de Consentimento Livre e Esclarecido (TCLE).

\section{Resultados}

O aplicativo móvel desenvolvido, recebeu o nome de “APH Mobile”, e está disponível nas lojas de aplicativos Google Play e Apple Store.

Por questões de segurança, ao inicializar o aplicativo é necessário informar login e senha de acesso. O profissional de saúde poderá acessar a listagem de pacientes já cadastrados ou cadastrar um novo paciente. A identificação é o início do relacionamento com o paciente. Cadastra-se o nome, idade, sexo, estado civil, RG, profissão, residência, se é alérgico, se possui alguma doença crônica e se faz uso de alguma medicação. No formulário de cadastro de pacientes, é possível vincular os dados 
do paciente com as equipes, assim a partir do momento que se vincula um paciente a uma equipe, todos os membros da equipe passam a visualizar todos os dados referentes ao paciente e também os atendimentos realizados. $\mathrm{O}$ aplicativo disponibiliza uma área, para que o próprio usuário faça os cadastros de equipes e membros. Concluindo o cadastro do paciente, pode-se iniciar o registro do atendimento.

O aplicativo móvel "APH Mobile" possui a tela para cadastro ou consulta de informações do paciente (formulários) e mais oito telas com as principais opções para registro das atividades assistenciais realizadas em um atendimento pré-hospitalar. A tela foi composta por oito ícones: motivo da solicitação; sinais vitais; história clínica; avaliação física; diagnóstico de enfermagem; prescrição de enfermagem; recusa de atendimento e destino, conforme Figura 1. As opções são:

- Motivo da solicitação: Registrar o motivo pela qual a equipe de atendimento pré-hospitalar foi acionada.

- Sinais vitais: Registro dos sinais vitais durante o atendimento pré-hospitalar, o qual foi subdivido em seis menus: pressão arterial, frequência respiratória, oximetria do pulso, temperatura, glicemia e dor. A inserção dos dados é realizada por meio de inserção das informações(formulário)

- História clínica: Registro da história clínica do paciente, separado pelos elementos da anamnese: queixa principal, história da doença atual, história médica pregressa, histórico familiar, história pessoal (fisiológica) e social.

- Avaliação física: Registro da avaliação física do paciente, o qual subdivido em seis telas: trato respiratório e coluna cervical, respiração e tórax, circulação, gastrointestinal, neurológico com escala de Glasgow e músculo esquelético.

Optou-se pelo desmembramento dos subitens baseado no protocolo ABCDE do trauma, o aplicativo também está preparado para registros de atendimento de casos clínicos. O método, desenvolvido por Jim Styner, é separado em cinco etapas: A, B, C, D e E. Cada letra representa uma etapa cuja inicial se refere a um termo em inglês. A de airway (via respiratória/trato respiratório); B de breathing (ou respiração); C de circulation (ou circulação); D de disability ou (ou incapacidade) e E de exposure (ou exposição). Cada subitem contém uma lista de opções para otimização do tempo do enfermeiro.

- Diagnóstico de enfermagem: Registro do diagnóstico de enfermagem.

- Prescrição de enfermagem: Registro da prescrição de enfermagem. Tanto o diagnóstico quanto a prescrição podem ser registrados por comando de voz com conversão em texto pelo aplicativo.

- Recusa ao atendimento: Registro da recusa do atendimento ou remoção com termo assinado pelo próprio paciente, testemunha ou responsável.

- Destino: Registro da remoção do paciente com informações do local, do nome da pessoa receptora e compartilhamento do atendimento prestado com a equipe que continuará com o atendimento.

Um detalhe importante é que se o usuário do aplicativo for um técnico de enfermagem, ele não visualiza as funcionalidades de diagnóstico e prescrição de enfermagem, para o técnico será apresentada a opção anotações e observações de enfermagem. 
Figura 1 - Telas do aplicativo para registro das atividades assistenciais realizadas em um atendimento pré-hospitalar.

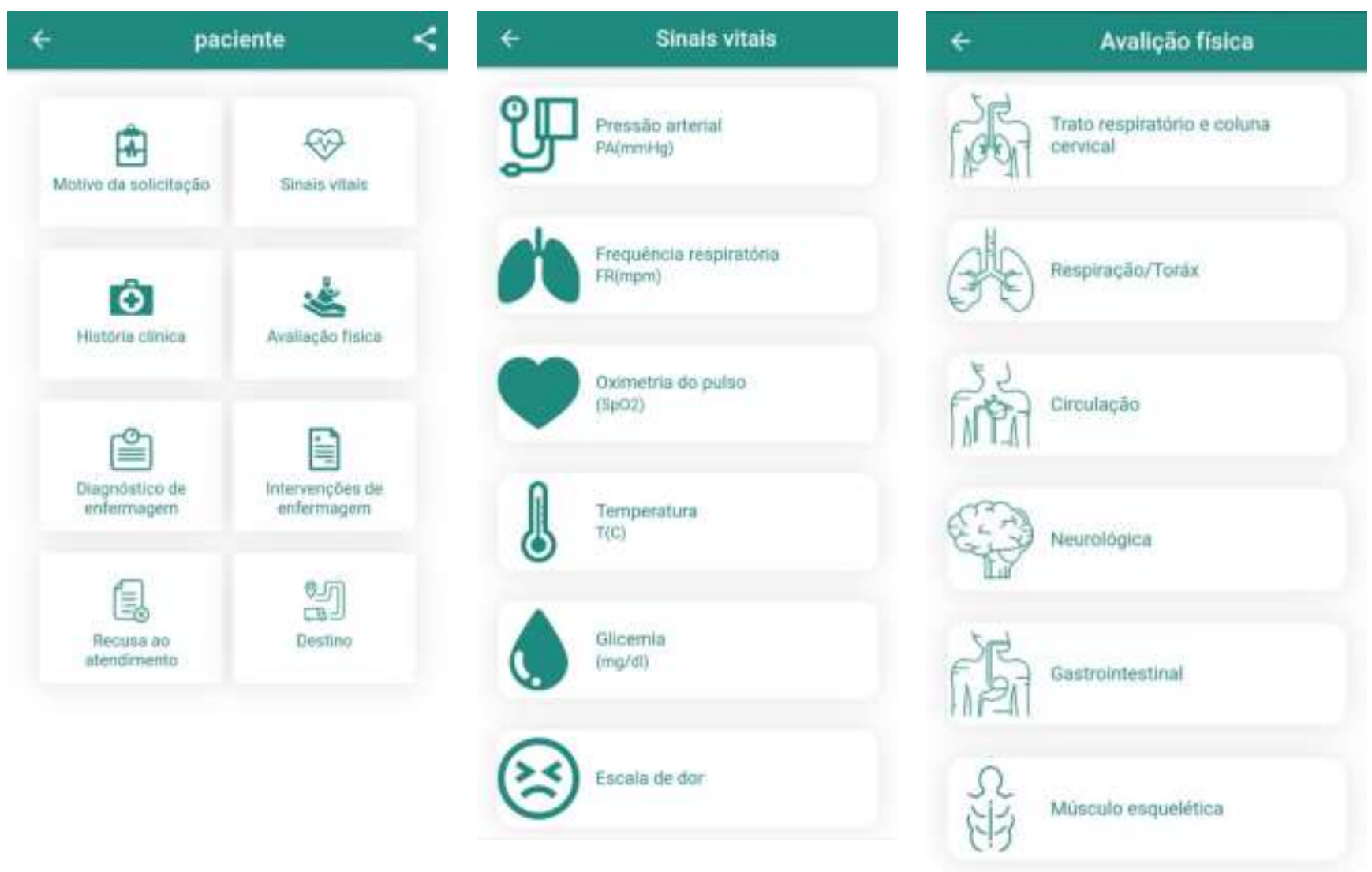

Fonte: Autores (2021).

Em relação aos participantes da pesquisa, quanto as características dos profissionais de atendimento pré-hospitalar, observaram-se que a maioria é do sexo feminino, atuando há mais cinco anos no atendimento pré-hospitalar e fizeram especialização. Quanto ao tempo de utilização de dispositivos móveis, observou-se que a maioria faz uso há mais de 5 anos e um pouco mais da metade passa entre 2 a 4 horas utilizando dispositivo móvel por dia. Em relação aos profissionais de tecnologia da informação, a maioria é do sexo masculino, atuando há mais de cinco anos na área. O Sistema Operacional mais usado no dispositivo móvel utilizado para realização dos testes pelos dois grupos de participantes foi o sistema Android.

Quanto a avaliação de usabilidade, SUS, segundo a avaliação por profissionais da saúde, a média obtida do escore total foi de 76,67 \pm 17,56 pontos; valor mínimo de 50 e máximo de 100 . Os participantes (8 e 13) pontuaram o aplicativo com o índice máximo de 100 e os participantes $(2,7,10,11$ e 15) pontuaram o aplicativo abaixo da média, segundo Índice SUS de 68 pontos (Figura 2). A menor pontuação foi 50 pontos, considerando mediano segundo a escala de classificação adjetiva. 
Figura 2 - Avaliação SUS por profissionais da saúde.

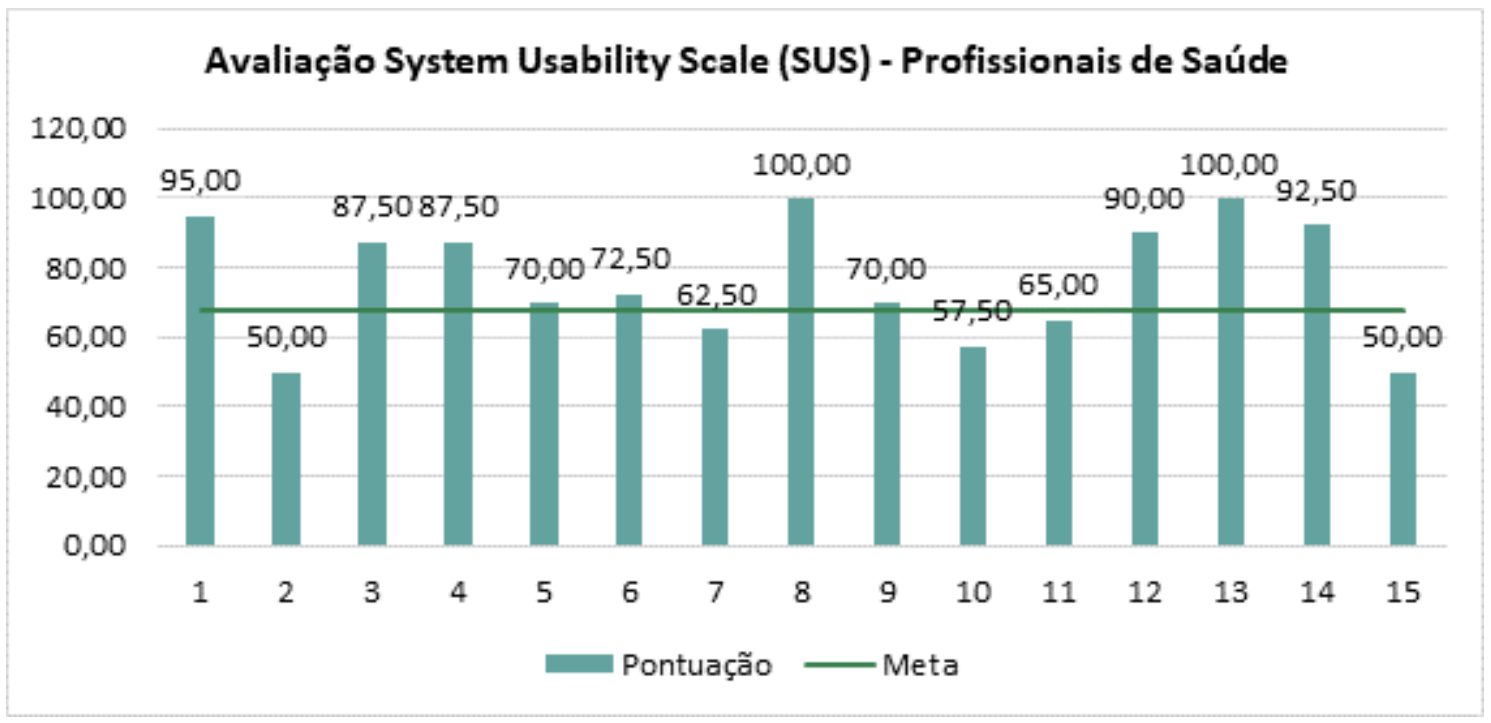

Fonte: Autores (2021).

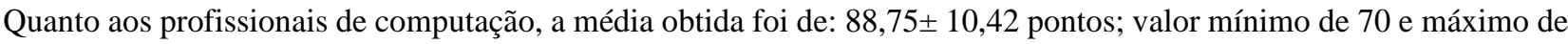
100. Quando analisada pelo teste de normalidade Shapiro-Wilk e teste estatístico Mann-Whitney, a associação do índice SUS com as variáveis sexo, idade, grau de escolaridade e área de atuação mostrou baixa correlação, não sendo estatisticamente significantes.

Figura 3 - Avaliação do SUS por profissionais de computação.

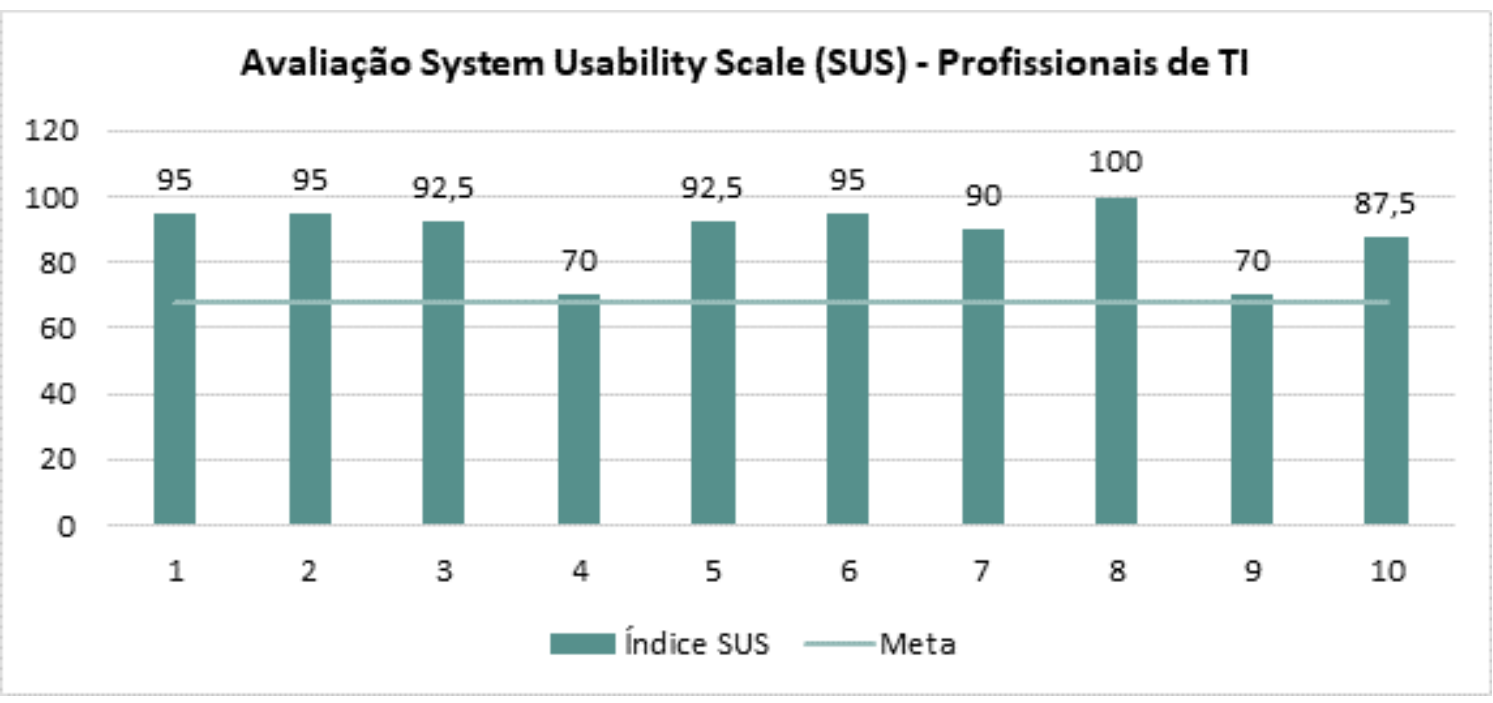

Fonte: Autores (2021).

Quando analisado, o aplicativo como um todo pelos dois grupos de participantes, chegamos à conclusão de que o aplicativo atende aos critérios de facilidade de aprendizagem, eficiência, minimização dos erros e satisfação do usuário. Identificamos que o critério facilidade de memorização precisa de ajustes, pois recebeu média de 66,67 pontos pelos profissionais de atendimento pré-hospitalar, um pouco abaixo da média de corte de 68 pontos. Essa pontuação foi a menor entre os critérios avaliados. Serão realizadas adequações no aplicativo para minimizar a carga de memória do usuário tornando os objetos, ações 
e opções visíveis. Destacamos ainda, que o critério satisfação do usuário foi o melhor pontuado pelos profissionais de atendimento pré-hospitalar, chegando a 84,44 pontos.

Os resultados obtidos pela avaliação do aplicativo, na presente pesquisa, auxiliarão a entender o comportamento, a opinião, os anseios e as necessidades dos usuários, podendo ser utilizado como material de apoio à decisão referente a possíveis alterações e/ou a atualizações dos recursos tecnológicos. A avaliação do aplicativo APH Mobile mostrou que os profissionais que utilizaram o sistema o consideram adequado, o que contribui para melhorar a confiabilidade na substituição do preenchimento em papel.

\section{Discussão}

Elaborar um aplicativo coerente e adequado é primordial, além de reconhecer as necessidades do público alvo, o desenvolvimento seja de acordo com os requisitos específicos, testados na pesquisa e implementados na prática. (Vescovi et.al, 2017; Padrini-Andrade et.al., 2019). O “APH Mobile” é uma inovação tecnológica na saúde, por ser um aplicativo que contém a maioria das funcionalidades para registro do atendimento pré-hospitalar que também foram levantadas em estudos similares que contemplam a utilização de aplicativos móveis para registro de atendimento pré-hospitalar, propondo o preenchimento automatizado das fichas de atendimento, visando diminuir a quantidade de campos preenchidos incompletamente ou erroneamente, por meio de um aplicativo (Gorini, 2018).

O aplicativo "APH Mobile”, atende as necessidades do profissional de saúde perante o registro do atendimento préhospitalar e vai além de apenas um formulário para preenchimento de informações, já que possibilita o acompanhamento em tempo real do quadro clínico do paciente pela equipe de regulação. $O$ aplicativo já foi desenvolvido de uma forma que permita o compartilhamento de dados com as equipes (regulação e/ou recebimento do paciente) para que essas, tenham conhecimento quase imediato dos sinais vitais, avaliação física e desfecho pela transmissão on-line dos dados do local do atendimento. É importante salientar que ao construir uma tecnologia deve ser levado em consideração a localização, pois ainda existem áreas (remotas ou rurais) desprovidas de acesso às tecnologias, seja por falta de acesso à rede de internet, ou diversos fatores (Aitken \& Lyle, 2015), por isso é preciso que o dispositivo também opere em modo off-line.

O instrumento SUS aplicado, foi eficiente para avaliar a usabilidade pela percepção do usuário, sendo classificado como bom (pontuação 76,57 \pm 17,57) demonstrando que o aplicativo atende exigências de usabilidade. Caso haja necessidade de uma identificação mais detalhada, outros instrumentos e métodos deverão ser utilizados. A avaliação do aplicativo "APH Mobile" mostrou que os profissionais que utilizaram o sistema o consideram adequado, o que contribui para melhorar a confiabilidade na substituição do preenchimento em papel.

Avanços tecnológicos possibilitam aos profissionais da saúde utilizar esses recursos dentro da prática, para melhorar a rotina, apoiar a decisão, otimizar o processo de registro, cuidado e avaliação, assumindo que quem possui informação adequada e atualizada, tem melhores condições de escolher e de tomar decisão (ITU, 2017). Vale salientar que a pesquisa apresentou limitação sobre a higiene das mãos, infecção e segurança do paciente, visto que os dispositivos móveis podem acarretar riscos de contaminação (COREN, 2016).

\section{Conclusões}

Foi desenvolvido um aplicativo móvel para registros de atendimento pré-hospitalar, avaliado quanto à satisfação do usuário e usabilidade. Com a avaliação preliminar possibilitou verificar a interação entre os usuários e o aplicativo. Outras avaliações de usabilidade serão realizadas num segundo momento, nesse ínterim, espera-se que este trabalho possa contribuir para o estabelecimento de um meio computacional como ferramenta para auxiliar as tomadas de decisão eficientes para a melhoria contínua do sistema de APH. 
Sugere-se que para trabalhos futuros acerca da temática, seja aplicado o questionário novamente para avaliação do aplicativo como um estudo piloto, com a implantação do aplicativo em um dispositivo móvel disponível em cada ambulância de APH, inclusive em ambulâncias de suporte avançados, para que não sejam mais utilizados formulários em papel e que a entrada de dados seja feita diretamente pelo profissional de APH na cena do evento ou durante o transporte da vítima. A fim de possibilitar analisar a percepção do uso do aplicativo pelos profissionais de saúde e evolução e melhorias no aplicativo.

\section{Referências}

Aitken, M., \& Lyle, J. (2015). Patient Adoption of mHealth. Use, Evidence and Remaining Barriers to Mainstream Acceptance. Parsippany: Institute for Healthcare Informatics. https://www.iqvia.com/-/media/iqvia/pdfs/institute-reports/patient-adoption-of-mhealth.pdf

Albuquerque, E. A. Y., Albuquerque, G. A., Souza, L. C., Santos, S. S., \& Rêgo, Y. L. S. (2017). Prontuário eletrônico do paciente e certificação de software em saúde: avanços que visam maior segurança dos dados médicos. Revista Brasileira de Inovação Tecnológica em Saúde, 7(2), 18-31. https://doi.org/10.18816/rbits.v7i2.11074

Bangor, A., Kortum, P. T., \& Miller, J. T. (2008). An Empirical Evaluation of the System Usability Scale. International Journal of Human-Computer Interaction, 24(6), 574-594. https://doi.org/10.1080/10447310802205776

Bangor, A., Kortum, P, \& Miller, J. (2009). Determining What Individual SUS Scores Mean: Adding an Adjective Rating Scale. Journal of Usability Studies, 4(3), 114-123. https://uxpajournal.org/wp-content/uploads/sites/7/pdf/JUS_Bangor_May2009.pdf

Barra, D. C. C., Paim, S. M. S., Sasso, G. T. M. D., \& Colla, G. W. (2018). Methods for developing mobile apps in health: na Integrative review of the literature. Texto \& Contexto - Enfermagem, 26(4), e2260017. https://doi.org/10.1590/0104-07072017002260017

Barros, B. C., Lima, K. B., Silva, Carmo, A. F. S., Moura, L. A. Barros, A. G., \& Korinfisky, J. P. (2014). O instrumento de registro do samu: com a palavra dos profissionais de saúde. Revista de Pesquisa Cuidado é Fundamental Online, 6(2), 618-626. https://doi.org/10.9789/2175-5361.2014v6n2p618

Bernardi, F. A., Senju, A L. A., Santos, M. A., Azevedo, B. M., \& Pereira Júnior, G. A. (2016) Dispositivo móvel para registro eletrônico multiprofissional do atendimento pré-hospitalar no SAMU. Journal of Health Informatics, 8(Suppl. 1), 879-888. https://docs.bvsalud.org/biblioref/2018/07/90666 2/anais_cbis_2016_artigos_completos-879-888.pdf

COREN. (2016). Parecer $n^{o}$ 005/CT/2016. Uso de aparelho celular no ambiente hospitalar. http://www.corensc.gov.br/wp-content/uploads/2016/08/ParecerT\%C3\%A9cnico-005-2016-Uso-de-aparelho-celular-no-ambiente-hospitalar.pdf

Gomes, M. L. S., Rodrigues, I. R., Moura, N. S., Bezerra, K. C., Lopes, B. B., Teixeira, J. J. D., \& Oriá, M. O. B. (2019). Evaluation of mobile Apps for health promotion of pregnant women with preeclampsia. Acta Paulista de Enfermagem, 32(3), 275-281. http://dx.doi.org/10.1590/1982-0194201900038

Gorini, I. M. (2018). Aplicação de BI voltado ao preenchimento digital da ficha de atendimento do SAMU, uma proposta para o back-end (Trabalho de conclusão de curso). UNICESUMAR, Maringá. http://rdu.unicesumar.edu.br/bitstream/123456789/635/1/Trabalho\%20de\%20Co nclus\%c3\%a3o\%20 de\%20Curso\%20TCC.pdf

ITU Telecommunication Development Sector (ITU-D) Study Groups. (2017). Question 5/1. Telecommunications/ICTs for rural and remote areas. Final Report. https://www.itu.int/dms_pub/itu-d/opb/stg/D-STG-SG01.05-2017-PDF-E.pdf

Lima, V. S., Lima, V. S., Vale, T. M., \& Pisa, I. T. (2018). Prontuário eletrônico do cidadão: desafios e superações no processo de informatização. Revista de Saúde Digital e Tecnologias Educacionais, 3, 100-113. http://www.periodicos.ufc.br/resdite/article/view/39756/95752

Nielsen, J. (1994). How to conduct a heuristic evaluation. Fremont: Nielsen Norman Group. https://www.nngroup.com/articles/how-to-conduct-a-heuristicevaluation/

Orlandi, C. (2018). Firebase: serviços, vantagens, quando utilizar e integrações [Blog]. https://blog.rocketseat.com.br/firebase/

Padrini-Andrade, L., Balda, R. C. X., Areco, K. C. N., Bandiera-Paiva, P., Nunes, M. V., Marba, S. T. M., \& Bomfim, O. (2019). Evaluation of usability of a neonatal health information system according to the user's perception. Revista Paulista de Pediatria, 37(1), 90-66. http://dx.doi.org/10.1590/1984$0462 / ; 2019 ; 37 ; 1 ; 00019$

Pereira A. S. et al. (2018). Metodologia da pesquisa científica. UFSM.

Pressman, R. S., \& Maxim, R. B. (2016). Engenharia de Software - uma Abordagem Profissional (8a ed). Bookman.

Portaria $n^{\circ}$ 288, de 12 de março de 2018. Redefine a operacionalização do cadastramento de serviços de atendimento pré-hospitalar móvel de urgência e o elenco de profissionais que compõem as equipes do Serviço de Atendimento Móvel de Urgência (SAMU 192) no Cadastro Nacional de Estabelecimentos de Saúde (CNES). https://bvsms.saude.gov.br/bvs/saudelegis/sas/2018/prt0288_29_03_2018.html

Ribeiro, J. C. (2017). Automação de Captura de Dados em Documentos de Atendimento Pré-Hospitalar: O Caso GSE Universidade Federal do Rio de Janeiro, http://www.itlab.poli.ufrj.br/pdf/monografias/2017/MBTi-e/automacao_de_captura_de_dados_em _documentos_pre_hospitalar.pdf

Santana, C. J., Oliveira, M. L. F., \& Marcon, S. S. (2019). Documentary analysis of patient's records: a systematic review of Literature. REPENF - Revista Paranaense de Enfermagem, 2(1), 75-84. http://seer.fafiman.br/index.php/REPEN/article/view/557/535 
Research, Society and Development, v. 10, n. 13, e463101321546, 2021

(CC BY 4.0) | ISSN 2525-3409 | DOI: http://dx.doi.org/10.33448/rsd-v10i13.21546

Tenório, J. M., Cohrs, F. M., Sdepanian, V. L., Pisa, I. T., \& Marin, H. D. F. (2011). Desenvolvimento e avaliação de um protocolo eletrônico para atendimento e monitoramento do paciente com doença celíaca. Revista de Informática Teórica e Aplicada, 17(2), 210-220. https://doi.org/10.22456/2175-2745.12119

Vêscovi, S. J. B., Primo, C. C., Sant'Anna, H. C., Bringuete, M. E. O., Rohr, R. V., Prado, T. N., \& Bicudo, S. D. S. (2017). Mobile application for evaluation of feet in people with diabetes mellitus. Acta Paulista de Enfermagem, 30(6), 607-613. http://dx.doi.org/10.1590/1982- 0194201700087 